\title{
ARCHIVE
}

Dr. Bernhard Homa, M.A.*

\section{Ausgewählte neuere Rechtsprechung zu archivischen Aufgabenfeldern: Kernaussagen und Schlussfolgerungen für die Praxis}

\section{Einleitung}

Die Bedeutung von Rechtsfragen für die archivarische Arbeitspraxis braucht heutzutage nicht mehr extra betont werden: Zahlreiche einschlägige Fachpublikationen, Tagungen und Fortbildungsveranstaltungen der letzten Jahre legen davon beredtes Zeugnis $a b^{1}$. Solche Fragen haben natürlich schon immer eine Rolle gespielt und entsprechende

* Der Autor ist Archivar am Niedersächsischen Landesarchiv Abteilung Hannover und Mitglied des Arbeitskreises „Archivische Rechtsfragen“ im VdA - Verband deutscher Archivarinnen und Archivare e.V. Die folgenden Ausführungen geben ausschließlich die persönlichen Auffassungen des Autors wieder.

1 Vgl. exemplarisch: RECHTsicher - Archive und ihr rechtlicher Rahmen. 89. Deutscher Archivtag in Suhl. Redaktion: Thomas Bardelle und Christian Helbich in Verbindung mit Eberhard Fritz, Ewald Grothe, Ulrike Gutzmann, Bettina Joergens, Michael Scholz, Kristina Starkloff. Fulda 2020 (Tagungsdokumentationen zum Deutschen Archivtag 24); Irmgard Christa Becker / Clemens Rehm / Udo Schäfer (Hg.), Nicht nur Archivgesetze ... Archivarinnen und Archivare auf schwankendem rechtlichem Boden? Best Practice - Kollisionen - Perspektiven. Beiträge zum 22. Archivwissenschaftlichen Kolloquium der Archivschule Marburg. Marburg 2019 (Veröffentlichungen der Archivschule Marburg 66); Archive im Rechtsstaat. Zwischen Rechtssicherung und Verrechtlichung. 51. Rheinischer Archivtag, 6.-7. Juli 2017 in Essen. Beiträge. Redaktion: Claudia Kauertz. Bonn 2018 (Archivhefte 49) [Fachthematische Tagung mit anschließender Publikation]; Irmgard Christa Becker / Clemens Rehm (Hg.), Archivrecht für die Praxis. Ein Handbuch. München 2017; Archive als Informationsdienstleister im Spannungsfeld zwischen Nutzerinteressen und Rechtsfragen. Referate des Landesarchivtags in Merseburg am 6./7. Mai 2015. Redaktion: Ralf Jacob. Fulda 2016; Jost Hausmann, Archivrecht. Ein Leitfaden. Frankfurt a.M. 2016; Alles was Recht ist. Archivische Fragen - juristische Antworten. 81. Deutscher Archivtag in Bremen. Red.: Heiner Schmitt. Fulda 2012 (Tagungsdokumentationen zum Deutschen Archivtag 16); sowie zuletzt auch Andreas NestL, Zugang im Archiv. Möglichkeiten und Grenzen für ein offenes Archiv im digitalen Zeitalter, in: RuZ - Recht und Zugang 1 (2020), S. 5-15 (DOI: https://doi.org/10.5771/2699-1284-2020-1-5). Einen guten systematischen Überblick bietet die Fachbibliographie der Archivschule Marburg, abrufbar unter: https://hds.hebis.de/asmr/in dex.php, zuletzt abgerufen am 04.06.2020. Zu archivrechtlichen Fortbildungen vgl. etwa das Fortbildungsprogramm 2020 der Archivschule Marburg, abrufbar unter: https://archivschule. 
Veröffentlichungen hervorgerufen²; zudem war auch die Zeit vor den ersten Archivgesetzen, wie zuletzt zurecht bemerkt worden ist, kein rechtsfreier Raum ${ }^{3}$. Dennoch kann mit gewisser Berechtigung festgestellt werden, dass das Bewusstsein für das Archivrecht - hier und im Folgenden verstanden als die Summe aller die archivarischen Fachaufgaben betreffenden juristischen Regelungen - in den letzten zehn bis fünfzehn Jahren nochmals stark gewachsen ist. Dies hängt vermutlich mit mehreren sich wechselseitig verstärkenden Entwicklungen zusammen ${ }^{4}$ :

1.) Dem verstärkten Transfer von Fachaufgaben wie zum Beispiel Überlieferungsbildung, Erschließung und Benutzung in die digitale Welt, parallel zu entsprechenden allgemeinen sozioökonomischen Entwicklungen.

2.) Einem sehr dynamisch fortschreitenden Verrechtlichungsprozess in verschiedenen archivrelevanten Rechtsbereichen wie dem Datenschutz- oder Urheberrecht sowie - erstere beide beeinflussend - dem Europarecht.

3.) Der Selbstwahrnehmung und dem Anspruch des archivarischen Berufsstandes als Wahrer rechtlicher Ansprüche und rechtskonformen Verhaltens in jedweder Form ${ }^{5}$.

de/uploads/Fortbildung/2020/Fobi_2019_V_12_WEB.PDF, zuletzt abgerufen am 04.06.2020, hier die Grundkurse 1201, 1202 und 1211, teilweise auch Aufbaukurs 5301.

$2 \mathrm{Vgl}$. die nach wie vor fundamentale Darstellung von Bartholomäus MANEGold, Archivrecht. Die Archivierungspflicht öffentlicher Stellen und das Archivzugangsrecht des historischen Forschers im Licht der Forschungsfreiheitsverbürgung des Art. 5 Abs. 3 GG. Berlin 2002 (Schriften zum Öffentlichen Recht 874); der aktuellste Überblick zur Archivgesetzgebung ist Hannes Berger, Öffentliche Archive und staatliches Wissen. Die Modernisierung des deutschen Archivrechts. Marburg 2019; weiter Petra NAU, Verfassungsrechtliche Anforderungen an Archivgesetze des Bundes und der Länder. Kiel 2000; Andreas NadLer, Die Archivierung und Benutzung staatlichen Archivgutes nach den Archivgesetzen des Bundes und der Länder. Diss. jur. Bonn 1995; zu einem schon vor den ersten Archivgesetzen virulenten Spezialthema: Harald MÜlLER, Rechtsprobleme bei Nachlässen in Bibliotheken und Archiven. Hamburg/Augsburg 1983 (Arbeitshefte der Arbeitsgemeinschaft für Juristisches Bibliotheks- und Dokumentationswesen 8). Für systematische Studien vgl. wiederum die Fachbibliographie der Archivschule (wie Anm. 1).

3 Vgl. Rainer Polley, Abschnitt II.2. Archivrecht in Deutschland, in: Becker / Rehm, Archivrecht für die Praxis (wie Anm. 1), S. 19-36, hier S. 19-27.

4 Diese Erkenntnis darf sicher als eine immer wieder geäußerte Feststellung der unter Anm. 1 genannten sowie weiterer jüngerer Publikationen gezogen werden; auf Einzelnachweise sei daher hier verzichtet.

5 So wird zum Beispiel im Berufsbild des VdA von 2009 unter anderem „Bewahrung der Rechte der Archivträger und der Bürger" als Auftrag der Archive definiert ([VdA], Das Berufsbild von Mitarbeiterinnen und Mitarbeitern in Archiven [2009], abrufbar unter: http://www.vda.ar chiv.net/fileadmin/user_upload/pdf/Arbeitskreise/Berufsbild/DasBerufsbild2009.pdf, zuletzt abgerufen am 04.06.2020. Solche Selbstbilder finden sich aber auch schon im Ethikkodex des Internationalen Archivrates von 1996, vgl. [ICA], Kodex ethischer Grundsätze für Archivarinnen und Archivare. 1996 (ursprünglich englisch: Code of Ethics), abrufbar unter: http://w ww.ica.org/sites/default/files/ICA_1996-09-06_code\%20of\%20ethics_DE.pdf, zuletzt abgerufen am 04.06.2020, hier Nr. 7. 
Dem letztgenannten Anspruch stehen allerdings erhebliche Hürden im praktischen Alltag gegenüber:

1.) Archivare sind üblicherweise keine Fachjuristen und schon von daher kaum in der Lage, alle archivrechtlich relevanten Rechtsgebiete zu überblicken oder juristische Methoden (Rechtsanwendung) präzise auf konkrete Sachverhalte umzumünzen.

2.) Handlungsleitende gerichtliche - erst recht ober- oder höchstgerichtliche - Entscheidungen mit konkretem Archivbezug sind insgesamt eher selten. Eine gewisse Ausnahme stellt hier die Häufung von Entscheidungen des Bundesverwaltungsgerichts aus den letzten Jahren zur Benutzung von Unterlagen der Nachrichtendienste dar, deren Tenor indirekt auch auf das Archivwesen insgesamt ausstrahlt. Analogieschlüsse aus verwandten Rechtsgebieten mit umfangreicherer Rechtsprechung (etwa dem Datenschutzrecht) sind zwar möglich und durchaus üblich, beinhalten aber ihre eigenen Probleme.

3.) Juristische Rechtsanwendung, auch wenn sie von allgemein formulierten Normen ausgeht, ist auf die Einzelfallabwägung fokussiert, die archivarische Arbeitspraxis jedoch ist auf das gleichförmige Massengeschäft ausgerichtet. Insbesondere im Bereich der Erschließung ist es aus praktischen Gründen meist schlicht unmöglich, einzelne Aktenteile blattweise auf rechtliche Probleme durchzusehen oder nach Ablauf von (Schutz-)Fristen eine nochmalige Prüfung - etwa auf noch bestehende Nutzungsversagungsgründe - vor der Freigabe für die Benutzung durchzuführen.

Insgesamt verwenden archivrechtliche Publikationen als Materialgrundlage den üblichen Mix aus der Kommentar- und sonstigen Literatur, den Gesetzesbegründungen, eigenen Gesetzesauslegungen sowie, soweit vorhanden, der Rechtsprechung. Gerade letztere sollte jedoch aus Sicht des Verfassers noch mehr Aufmerksamkeit erhalten, weil sie einerseits Auslegungsfragen klären helfen, andererseits zur Stützung eigener Positionen im Kontakt mit weiteren Akteuren benutzt werden kann.

Eine aktuelle spezifische Sammlung archivrelevanter Rechtsprechung ist dem Verfasser nicht bekannt, sieht man einmal von der sehr verdienstvollen, von 1958 bis 2014 reichenden Sammlung von Dieter Strauch ab, die aktuell unter der alten Adresse des von Klaus Graf geführten Blogs „Archivalia“ gesichert wird". Allerdings ist auch Strauchs Entscheidungssammlung - was angesichts der geschilderten disparaten Lage wenig verwunderlich ist - keineswegs vollständig.

Im Folgenden sollen einige aus Sicht des Verfassers relevante jüngere Gerichtsentscheidungen unter Berücksichtigung oben genannter Probleme dargestellt werden. Im Vordergrund stehen dabei folgende Leitfragen:

6 Aufgeteilt in drei Teile: 1.) https://archiv.twoday.net/stories/948994228, 2.) https://archiv.tw oday.net/stories/948994229, 3.) http://archiv.twoday.net/stories/948994230, jeweils abgerufen am 04.06.2020. 
1.) Welche Rückwirkungen sind für die infrage stehenden Fachaufgaben daraus abzuleiten?

2.) Welche Folgerungen ergeben sich daraus insgesamt für den Umgang mit Rechtsfragen und Rechtsanwendung in der archivischen Praxis?

Es geht also ausdrücklich nicht um eine juristische Dogmatik oder Systematik für diese ist der Verfasser nicht kompetent und die Entscheidungssammlung nicht vollständig genug -, sondern um einige aus dem Vergleich von Arbeitspraxis und Gerichtsentscheidungen gewonnene Wahrnehmungen. Die im Folgenden besprochenen Entscheidungen werden daher auch nicht detailliert diskutiert. Da deren Gehalt zudem meist mehrere Fachaufgaben betrifft, sind gewisse Redundanzen nicht zu vermeiden. In einem Anhang erfolgt eine kurze Synopse der besprochenen Entscheidungen

\section{Benutzung}

1. Grenzen des Persönlichkeitsrechts: Veröffentlichung personenbezogener Angaben und Rechtsgüterabwägung

Das Bundesverfassungsgericht hat sich im November 2019 in zwei auch öffentlich weithin rezipierten Entscheidungen mit der Thematik der Veröffentlichung und Auffindbarkeit personenbezogener Informationen im Internet auseinandergesetzt („Recht auf Vergessen" I und II $)^{7}$. Auch wenn sich die Beschlüsse konkret mit Onlinearchiven von Presseunternehmen befassen, sind die Kernaussagen aus Sicht des Verfassers auf Archive generell übertragbar:

1.) (Online-)Archive sind im öffentlichen Interesse, sie dienen der Bildung und Erziehung sowie der Debatte in der Demokratie ${ }^{9}$ - die gerne auf Archivtags-Festreden verwendeten Schlagworte von der Sicherungsfunktion und Systemrelevanz der Archive in der Demokratie werden damit auch verfassungsgerichtlich bestätigt.

2.) Damit zusammenhängend ist eine Löschung oder Vernichtung von Berichten aus Archiven mit Art. 5 Abs. 1 Satz 2 GG (Pressefreiheit) unvereinbar - gleiches ist per Analogie für Art. 5 Abs. 3 (Wissenschaftsfreiheit) anzunehmen.

3.) Ein pauschaler Anspruch auf Nicht-Veröffentlichung personenbezogener Daten noch Lebender existiert nicht.

7 BVerfG, Beschl. v. 06.11.2019 - 1 BvR 16/13 (vgl. für einen Folgebeschluss: https://archivali a.hypotheses.org/122895, zuletzt abgerufen am 06.06.2020 und BVerfG, Beschl. v. 06.11.2019 - 1 BvR 276/17. Die hier aufgezeigte Grundtendenz ist in weiteren Entscheidungen bestätigt worden, vgl. etwa BVerfG, Beschl. v. 07.07.2020 - 1 BvR 146/17; BGH, Urt. v. 27.07.2020 - VI ZR 405/18.

8 Die ebenfalls in beiden Entscheidungen behandelten Fragen bezüglich des Verhältnisses zwischen nationalem Verfassungs- und europäischem Unionsrecht werden hier nicht erörtert.

9 BVerfG, Beschl. v. 06.11.2019 - 1 BvR 16/13, Rn. 113. Dass es hier konkret um (private) Onlinearchive geht, ändert nach Ansicht des Verfassers nichts an der grundsätzlich auf alle, digitalen wie analogen Archive übertragbaren Grundaussage. 
4.) Es gibt keine Verpflichtung zu proaktiven Schutzmaßnahmen und regelmäßigen Überprüfungen, ob vormals rechtmäßig veröffentlichte personenbezogene Daten immer noch rechtmäßig online verbreitet werden.

Insgesamt hat das Bundesverfassungsgericht die Position von Archiven damit indirekt deutlich gestärkt: Für die Nr. 1.) und 2.) gilt dies zunächst abstrakt hinsichtlich der gesellschaftlichen Rolle der Archive, wobei die Nr. 2.) mittelbar durchaus mit der Problematik des Verhältnisses der Anbietungspflicht zu gesetzlichen Löschungs- und Vernichtungsregelungen in Verbindung gebracht werden $\operatorname{kann}^{10}$ : diese sind verfassungsrechtlich demnach eher kritisch zu bewerten. Nr. 3.) und 4.) sind jedenfalls praktisch im Hinblick auf Zugänglichmachung und Weiterverwendung von Archivgut relevant: Einerseits wird damit deutlich, dass es eben auch im Internetzeitalter keinen Vorrang des allgemeinen Persönlichkeitsrechts (Äußerungsrecht, informationelle Selbstbestimmung) gegenüber konkurrierenden Grundrechtspositionen gibt. Die Rechtsgüterabwägung - etwa im Rahmen von Schutzfristenverkürzungen - hat diese grundsätzliche Gleichwertigkeit zu berücksichtigen ${ }^{11}$. Einen automatischen Zwang zur Anonymisierung - obwohl als klassische Auflage in vielen Archivgesetzen und Benutzungsordnungen genannt ${ }^{12}$ - gibt es insofern ebenso wenig wie die Notwendigkeit, jedwede Beeinträchtigung schutzwürdiger Belange auszuschließen, was den archivarischen Ermessensspielraum erweitert ${ }^{13}$. Zum anderen werden derartigen Prüfpflichten deutliche Grenzen gesetzt: So können zum Beispiel nach Ablauf von Schutzfristen personenbezogene Daten im Regelfall bedenkenlos veröffentlicht werden, auch wenn es, wie im-

10 Vgl. dazu weiter unten Abschnitt IV.

$11 \mathrm{Zu}$ dieser Materie vgl. etwa auch die konzisen Überblicke von Michael KLeIN, Vom Umgang mit schwierigen Unterlagen: Personenbezogenes Archivgut in der Benutzung, in: Marcus Stumpf / Katharina Tiemann (Hg.), „Im (virtuellen) Lesesaal ist für Sie ein Platz reserviert ...". Archivbenutzung heute - Perspektiven für morgen. Beiträge des 21. Fortbildungsseminars der Bundeskonferenz der Kommunalarchive (BKK) in Kassel vom 14. - 16. November 2012. Münster 2013 (Texte und Untersuchungen zur Archivpflege 27), S. 53-64; Johannes Beleites, Zwei Grundrechte im Streit. Das Archivrecht im Spannungsverhältnis von Datenschutz und Informationsfreiheit. Vortrag gehalten auf dem 2. Workshop Archiv- und Bibliothekswesen vom 09.06 - 18.06.2006 in Kronstadt am 14. Juni 2006, abrufbar unter: http://www.honterus-archiv.ro/fileadmin/user_upload/pdfs/14-7-06_Beleites.2_Grundrecht e_im_Streit.net.pdf, zuletzt abgerufen am 25.03.2016, der allerdings durchaus einen latenten Vorrang des Datenschutzes konstatiert.

12 Vgl. z.B. $\$ 12$ Abs. 2 Satz 2 Nr. 2 BArchG; $\$ 6$ Abs. 4 Satz 3 LArchG BW.

13 Vgl. zur Forderung, den diesbezüglichen Ermessensspielraum auch auszunutzen - hier bezüglich der Reproduktionen aus Archivgut - Ehrhart KÖRTING, Welche Beschränkungen lässt das Archivrecht bei wissenschaftlichen Forschungsvorhaben zu national-sozialistischem Massenmord zu? (Blogbeitrag vom 18.08.2019, abrufbar unter: https://www.gedenkort-t4.eu /de/blog/welche-beschraenkungen-laesst-das-archivrecht-bei-wissenschaftlichen-forschungs vorhaben-zu, zuletzt abgerufen am 21.08.2019); Vinzenz LÜBBEN, Stolperfallen im Netz. Postmortaler Persönlichkeitsschutz und die Belange von Hinterbliebenen, in: BECKER u.a., Nicht nur Archivgesetze (wie Anm. 1), S. 151-169, hier S. 157f., hält dagegen einen solchen völligen Ausschluss der Beeinträchtigung schutzwürdiger Belange bei Publikationen im Internet tendenziell für notwendig. 
mer, Ausnahmen geben wird ${ }^{14}$ - selbst wenn diese Fristen sich nachher als falsch herausstellen, etwa wegen unbekannter/fehlerhafter Lebensdaten, handelt es sich hier gerade nicht um einen Rechtsverstoß und erfordert unter Umständen noch nicht einmal deren Entfernung ${ }^{15}$. Zur regelmäßigen Nachprüfung der weiterhin bestehenden Rechtmäßigkeit ist niemand verpflichtet. Diese Einschränkung des allgemeinen Persönlichkeitsrechts auch im Internetzeitalter begründet das Bundesverfassungsgericht eben mit dem unter 1.) genannten öffentlichen Interesse an der Existenz und Zugänglichkeit von Archiven - insofern ist dieser Punkt eben nicht nur abstrakt, sondern auch praxisrelevant.

Erkennbar wird an beiden Entscheidungen allerdings auch die weiter oben bemerkte Problematik der Analogieschlüsse, denn:

1.) Die Beklagten waren ein Presseunternehmen und ein Suchmaschinenbetreiber und damit Grundrechtsträger, während sich das Informationshandeln öffentlicher Archive darauf bestenfalls indirekt (Grundrechte der Benutzer, Art. 5 Abs. 1 und 3 GG) berufen kann.

2.) Die Thematik „Zeitablauf“ wird jedenfalls in diesem Zusammenhang diametral anders als im Archivwesen eingeschätzt. Denn während im Archivwesen üblicherweise mit dem Zeitablauf von einem allmählichen Nachlassen der Schutzwürdigkeit ausgegangen wird, sodass schon 30- oder 60-jährige Schutzfristen eine Auffangfunktion übernehmen können ${ }^{16}$, hebt das Bundesverfassungsgericht gerade umgekehrt darauf ab, dass - lebende! - Betroffene bei fortschreitender Dauer immer stärkeren Anspruch darauf haben, nicht mehr mit früheren Handlungen konfrontiert zu werden. Ob dies zum Beispiel im Falle der Veröffentlichung archivischer Erschließungsinformationen nach Ablauf archivgesetzlicher Schutzfristen anders zu bewerten wäre, kann jedenfalls auf dieser Grundlage nicht abschließend geklärt werden.

Letztlich ist also die Frage der Anwendbarkeit für die archivrechtliche Praxis davon abhängig, ob und wenn ja in welcher Auslegung man eine Übertragbarkeit auf die eigenen Fallkonstellationen für möglich hält.

\section{Nutzungsversagung und postmortales Persönlichkeitsrecht}

Ein etwa in Bezug auf Unterlagen zu NS-Verbrechen oder Unterlagen mit medizinischen Angaben immer wieder diskutiertes Thema ist die Frage des „postmortalen Persönlichkeitsrechtes“ und damit zusammenhängend der Nutzungsversagung auch nach

$14 \mathrm{Zu}$ Einschränkungen vgl. die Einschätzung von Nestr, Zugang im Archiv (wie Anm. 1), S. 9 sowie Abschnitt 2. dieses Aufsatzes.

15 Dass hier im Fall der Fälle je nach Materie und Schutzwürdigkeit (Sozial-, Privat- und Intimsphäre) unterschiedlich zu entscheiden wäre, versteht sich.

16 Vgl. Klein, Personenbezogenes Archivgut in der Benutzung (wie Anm. 11), hier S. 55f., 58.

RuZ 2. Jg. 3/2021 
Ablauf von Schutzfristen wegen schutzwürdiger Interessen Betroffener oder Dritter, insbesondere Angehöriger ${ }^{17}$.

Mit diesen Fragen hat sich nun auch das Bundesverwaltungsgericht, unter Zugrundelegung des Bundesarchivgesetzes, zuletzt in einer Reihe von Entscheidungen im Rahmen des von Pressevertretern begehrten Zugangs zu Unterlagen der Nachrichtendienste beschäftigt ${ }^{18}$. Die - freilich schon früher in der Rechtsprechung formulierten Kernaussagen lauten wie folgt:

1.) Pauschalisierende Begründungen zur Nutzungsversagung aufgrund schutzwürdiger Belange Dritter sind unzulässig: diese müssen immer konkret und nachvollziehbar begründet werden.

2.) Das allgemeine Persönlichkeitsrecht endet mit dem Tod und der allgemeine Achtungsanspruch (= postmortales Persönlichkeitsrecht) schützt Verstorbene vor grober Herabwürdigung und Erniedrigung sowie deren Lebensleistung, doch die Veröffentlichung wahrer Tatsachenangaben über einen Verstorbenen verletzt seine Menschenwürde grundsätzlich nie.

Auch diese Entscheidungen stärken im Endeffekt sowohl die Position der Archive als auch die Möglichkeiten zur Benutzung und Zugänglichmachung, etwa die Digitalisierungen von Beständen ${ }^{19}$.

17 Vgl. exemplarisch die gelungene Problematisierung bei LübBEN, Stolperfallen (wie Anm. 13), S. 158-165; weiter Ehrhart KöRTING, Persönlichkeitsrecht und Archivrecht, in: Archive im Rechtsstaat (wie Anm. 1), S. 126-131; Mark Alexander STEINERT, Stichtag 19. Februar: Zehn Jahre Novelle des Personenstandsgesetzes. Rechtliche und praktische Fragen zur Archivierung von Personenstandsregistern, in: Archivar 70/1 (2017); Arnd Vollmer, Aktuelle rechtliche Fragen der Archivbenutzung, in: [Landesverband Sachsen im Verband deutscher Archivarinnen und Archivare e.V / Sächsisches Staatsarchiv (Hg.)], Ist der Kunde König? Was Benutzer wollen und Archive leisten können. 18. Sächsischer Archivtag 24.-26. Juni 2011 in Görlitz, Chemnitz 2012, abrufbar unter https://www.vda.lvsachsen.archiv.net/fileadmin/use r_upload/2011_goerlitz.pdf zuletzt abgerufen am 17.09.2019, S. 29-37, hier S.29-33; als Überblick zur Thematik: Jenny KотTE, VI.2.3. Einschränkung und Versagung der Nutzung, in: BecKer / ReHM, Archivrecht (wie Anm. 1), S. 165-170; bezüglich des BArchG: Christoph ParTsCH (Hg.), Bundesarchivgesetz. Handkommentar. Baden-Baden 2019, hier S. 225-238 (zu \13 BArchG). Vgl. jetzt auch Axel MeTz, Die Rechte der Nachkommen - oder: Schutz jenseits der Schutzfristen und die Konsequenzen für die Benutzung von Archivalien, in: RECHTsicher - Archive und ihr rechtlicher Rahmen (wie Anm. 1), S. 157-166.

18 Im Folgenden nach BVerwG, Beschl. v. 08.02.2019 - 20 F 2/17, sowie BVerwG, Urt. v. 30.01.2019 - 6 A 1/17; vgl. weiter auch BVerwG, Beschl. v. 20.12.2016 - 20 F 10/15, weitere Entscheidungen mit ähnlichem Tenor liegen vor; vgl. dazu auch PARTSCH, Bundesarchivgesetz. Handkommentar (wie Anm. 17), hier S. 225-238 (zu \$13 BArchG) und insbesondere bereits Manegold, Archivrecht (wie Anm. 2), S. 348-351. Beklagte sind in diesen Fällen die Behörden, da die Unterlagen noch bei diesen und nicht im Bundesarchiv lagern. Im Grundsatz ähnlich, aber mit Bezug auf ein parlamentarisches Auskunftsersuchen: BVerfG, Beschl. v. 13.06.2017 - $2 \mathrm{BvE} 1 / 15$.

19 Im Hinblick auf diese Rechtsprechung daher ausdrücklich abzulehnen ist die von Kai Engelbrecht, Gläserne Abstammung? - Zur digitalen Publikation archivierter Personenstandsregister in der Ordnung des Grundgesetzes, in: Die Öffentliche Verwaltung 70 (2017), S. 393-403, vertretene Haltung einer Unzulässigkeit der Digitalisierung wegen theoretisch möglicher - aber eben nicht konkret verifizierter - Beeinträchtigung schutzwürdiger Belange 
Allerdings kann man natürlich weiterhin diskutieren, was unter „wahren Tatsachenangaben“ zu verstehen ist: Wäre zum Beispiel die Veröffentlichung im Internet von Unterlagen in diffamierender NS-Sprache über die Opfer der NS-Krankenmorde zulässig oder nicht, präziser: muss auf den inbaltlichen oder den kontextuellen Wahrheitsgehalt (= die Existenz solcher Akten und von deren Inhalten ist wahr, mag dieser Inhalt auch menschenverachtend und insofern unwahr sein) abgestellt werden ${ }^{20}$ ? Letztlich werden Archive auch weiterhin vor solche Abwägungsentscheidungen gestellt werden, die nie und nirgends durch eine endgültige Rechtsprechung geklärt werden können. Doch mag in schärfster Variante durchaus die Linie vertreten werden, dass Veröffentlichungen von Archivgut niemals das postmortale Persönlichkeitsrecht verletzen. In dieser Hinsicht kann nur auf die oben genannte Kernaussage verwiesen werden: Egal wie entschieden wurde, dies muss immer konkret und nachvollziehbar begründet - und also auch dokumentiert! - werden.

Für Archive stellt sich außerdem die drängende Frage, inwiefern bei der Benutzung von Unterlagen auch nach Ablauf von Schutzfristen aktiv nach Nutzungsversagungsgründen nachgeforscht werden muss. Die Literatur beurteilt diese Frage unterschiedlich $^{21}$, während die Gesetzesbegründung zum BArchG von 2017 tendenziell in Richtung einer solchen proaktiven Ermittlungspflicht geht ${ }^{22}$. Letztere ist allerdings schlichtweg praxisfremd und undurchführbar: sie würde konsequent zu Ende gedacht nämlich bedeuten, sämtliche - und zwar wirklich sämtliche, also nicht nur personen-

heute Lebender. Geradezu paradox ist übrigens, dass das von Engelbrecht selbst verfassungsgerichtlich begründete „Recht auf Kenntnis der eigenen Abstammung“ (ebd. S. 394f.) vielmehr für statt gegen eine Digitalisierung der Register spricht; zum dahinter stehenden Fall aus Bayern insgesamt NeSTL, Zugang im Archiv (wie Anm. 1), S. 7-10; konzise in der Ablehnung pauschalisierender Behauptungen seitens des Datenschutzbeauftragten in NRW LÜBBEN, Stolperfallen (wie Anm. 13), S. 162-165.

20 Ehrhart KöRTING, Die Wiederherstellung des „Personseins“ von Opfern der NS-„Euthanasie“ von 1939 bis 1945, in: Andreas Nachama / Uwe Neumärker (Hg.), Gedenken und Datenschutz. Die öffentliche Nennung der Namen von NS-Opfern in Ausstellungen, Gedenkbüchern und Datenbanken. Berlin 2017 (Stiftung Topographie des Terrors. Notizen. Bd. 12), S. 19-30 plädiert zwar grundsätzlich für eine veröffentlichungsfreundliche Auslegung des postmortalen Persönlichkeitsrechts, sieht aber etwa bei der Darstellung von Krankheitsgeschichte, geistigen und körperlichen Behinderungen, in der Familie vorhandener „Erbkrankheiten“" oder deren entwürdigenden Behandlung durch die Nationalsozialisten Ausschlussgründe auch nach Ablauf von Schutzfristen.

21 Bejahend etwa KотTE, Einschränkung und Versagung der Nutzung (wie Anm. 17), S. 165f.; vorsichtiger MANEGOLD, Archivrecht (wie Anm. 2), S. 346 mit Anm. 692.

22 Bundestagsdrucksache 18/9633 vom 15.09.2016, abrufbar unter https://dip21.bundestag.de/d ip21/btd/18/096/1809633.pdf, zuletzt abgerufen am 08.06.2020, hier S. 68: „, [...] dass das Bundesarchiv in diesen wie auch in allen anderen Benutzungsfällen gemäß $\$ 13$ Absatz 1 Nummer 2 prüfen muss, ob Grund zu der Annahme besteht, dass schutzwürdige Interessen Betroffener entgegenstehen". Nebenbei bemerkt, kommt es hier auch auf die Gesetzesformulierungen an: Während es z.B. in $\ 13$ Abs. 1 BArchG heißt „Das Bundesarchiv hat die Nutzung nach den $\mathbb{S} 10$ bis 12 einzuschränken oder zu versagen, wenn [...]“ ist die Nutzungsversagung in $\$ 5$ Abs. 4 NArchG nur als Kann-Bestimmung ausgeführt. Zudem sah der niedersächsische Landesgesetzgeber gerade keine aktive Ermittlungspflicht der öffentlichen Archive, vgl. Niedersächsischer Landtag, Drucksache 12/4271, hier S. $18 f$. 
bezogene! - Akten ab einem gewissen Stichdatum vor der Benutzung Blatt für Blatt darauf hin durchzusehen. Und werden schutzwürdige Belange festgestellt, wird es erst recht aufwendig: Denn das Bundesverwaltungsgericht hat zwar festgestellt, dass, da die Nutzungsversagungsgründe vom Schutzniveau her unter demjenigen der personenbezogenen Schutzfristen stehen, hier nur eine geringere Schutzfrist von 90 Jahren nach Geburt der infrage stehenden Personen angesetzt werden muss, jedoch erst nach einer Melderegisterabfrage, Internet- und Datenbankrecherchen und Nachfrage bei anderen Behörden ${ }^{23}$. Die juristische Norm und Rechtsprechung ist hier also mit der archivischen massenhaften Alltagspraxis tendenziell inkompatibel beziehungsweise letztere für erstere nicht berücksichtigt worden. Zugleich muss aber festgehalten werden, dass eine solche massenhafte Prüfpflicht selbst wiederum dem gesetzlich wie richterlich festgelegten Grundsatz der Vermeidung eines unverhältnismäßigen Verwaltungsaufwandes zuwiderläuft ${ }^{24}$. Rein praktisch gesehen bestehen zudem angesichts der restriktiven Auslegung des Bundesverwaltungsgerichts, wann überhaupt schutzwürdige Belange anzunehmen sind, für etwaige Beschwerden Betroffener oder Dritter hohe Hürden in der Begründung. Kombiniert mit den weiter oben zitierten Entscheidungen des Bundesverfassungsgerichts darf man zumindest für einen Großteil des jüngeren Archivguts annehmen, dass hier nach Ablauf von Schutzfristen eben keine aktive Ermittlungspflicht besteht. Vom Arbeitsablauf ist es daher sicher sinnvoll:

1.) Unterlagentypen zu definieren, bei denen solche schutzwürdigen Belange potenziell anzutreffen sind und diese einer entsprechenden Prüfung zu unterziehen ${ }^{25}$;

2.) diese Prüfung möglichst bereits im Rahmen der Erschließung durchzuführen und die getroffenen Entscheidungen - zum Beispiel verlängerte Schutzfristen - nachvollziehbar zu dokumentieren ${ }^{26}$. Denn bei womöglich Jahrzehnte später gestellten Benutzungsanträgen ist dies einerseits nicht praktikabel machbar, andererseits muss bei zwischenzeitlich womöglich geänderter Rechtslage erkennbar bleiben, mit welcher Begründung vorige Entscheidungen gefällt wurden.

23 BVerwG, Urt. v. 30.01.2019 - 6 A 1/17, hier Rn. 45-46.

$24 \mathrm{Vgl}$. etwa - ironischerweise wiederum auf die Nutzungsversagung bezogen $-\$ 13$ Abs. 2 Nr. 2 BArchG; restriktiv für die Annahme eines solchen Ausschlussgrundes, jedoch sehr wohl eine Grenze bei zu großer Menge an Unterlagen und im Hinblick auf die zur Verfügung stehenden (Personal-)Mittel ziehend: BVerwG, Urt. v. 17.03.2016 - 7 C 2/15 (hier bezogen auf das IFG).

25 Der „berühmt-berüchtigte“ Klassiker sind hier sicherlich staatsanwaltschaftliche Ermittlungsakten zu Sexualdelikten.

26 Eine ähnliche Vorgehensweise schlägt Johannes RosenplänTER, Menschenleere Strände. Das Fotoarchiv Online des Stadtarchivs Kiel und das Recht am eigenen Bild, in: Marcus STUMPF / Katharina Tiemann (Hg.), Fotos und Filme im Archiv - von analog bis digital. Beiträge des 25. Fortbildungsseminars der Bundeskonferenz der Kommunalarchive (BKK) in Erfurt vom 23.-25. November 2016. Münster 2017 (Texte und Untersuchungen zur Archivpflege 33), abrufbar unter: http://www.lwl.org/waa-download/publikationen/TUA_33.pdf, zuletzt abgerufen am 28.01.2018, S. 112-127, hier S. 123, für die Prüfung übernommener Fotomaterialien im Hinblick auf schutzwürdige personenbezogene Darstellungen vor. 
Auch in diesem Fall müssen Archive also die am Einzelfall orientierte Rechtsprechung für die archivische Massenpraxis kompatibel machen - derartige Transferleistungen kann und wird einem kein Gericht oder Gesetz abnehmen können.

\section{Urheberrecht und Archivrecht}

„Das Urheberrechtsgesetz ist nicht dazu gedacht, das Leben der Menschen einfacher zu machen“, wurde der Verfasser während der Ausbildung belehrt - noch treffender wäre wohl: Es ist dazu gedacht, den Archiven das Leben maximal gruselig zu machen, wie man wohl als Quintessenz die entsprechend reichhaltige Literatur zur Thematik zusammenfassen darf ${ }^{27}$. Als wesentliche Kernprobleme lassen sich festhalten:

1.) Das Urheberrecht ist in seiner ganzen Anlage auf das Einzelstück und den Schutz der ökonomischen wie ideellen Rechte von dessen Urheber angelegt, diese Zielstellung überwiegt in der Gewichtung bei weitem die - etwa in den Schrankenregelungen zusammengefassten - Rechte der Benutzer. Mit der archivischen gleichförmigen Erschließungs- und Benutzungspraxis ist dies inkompatibel.

27 Vgl. dazu und zum Folgenden etwa Neste, Zugang im Archiv (wie Anm. 1), hier S. 10-12; Thomas KRÄMER / Alexandra Zilles, Urheberrecht als archivische Herausforderung. Zum Umgang mit rechtlichen und technischen Dynamiken, in: Archive im Rechtsstaat (wie Anm. 1), S. 105-118; [KLA], Die bisherigen Reformen des Urheberrechts und die Onlinestellung digitalisierten oder digitalen Archivguts. [2019], abrufbar unter: https://www.bunde sarchiv.de/DE/Content/Downloads/KLA/aur-gutachten-onlinestellung-digitalisate.pdf?_b lob=publicationFile, zuletzt abgerufen am 12.11.2019; Katrin BEYER, Urheberrechtliche Fragen bei der Erschließung, Benutzung und Präsentation am Beispiel von Nachlässen im Geheimen Staatsarchiv Preußischer Kulturbesitz, in: Irmgard Christa BECKER / Valeska KOAL (Hg.), Archivisches Handeln. Strategien und Perspektiven unter dem Einfluss neuer Technologien. Marburg 2017 (Veröffentlichungen der Archivschule Marburg 62), S. 11-42; Mark Alexander STEINERT, Urheberrechtliche Probleme bei der Zugänglichmachung und Benutzung von Archivgut, in: Archive in Bayern 8 (2014), S. 231-247; Ders., Digitalisierung und Digitalisate im Lesesaal - urheberrechtliche Fragestellungen, in: STUmPF / TiEmann (Hg.), Archivbenutzung heute (wie Anm. 11), S. 65-74; Rainer Polley, Aspekte des Urheberrechts bei archivischen Nachlässen, in: Eva-Marie Felschow / Katharina Schaal (Hg.), Persönlichkeitsschutz in Archiven der Hochschulen und wissenschaftlichen Institutionen. Frühjahrstagung der Fachgruppe 8 im Verband Deutscher Archivarinnen und Archivare e.V., 21. bis 23. März 2012 an den Universitäten Gießen und Marburg. Leipzig 2013 (Wissenschaftsarchive 2), S. 134-152; Regina Rousavy, Mit Mut und Umsicht - Aspekte des Umgangs mit dem Urheberrecht bei nichtamtlichem Archivgut, in: Marcus STUMPF / Katharina TiEmanN (Hg.), Nichtamtliches Archivgut in Kommunalarchiven. Teil 2: Bestandserhaltung, Dokumentationsprofil, Rechtsfragen. Beiträge des 20. Fortbildungsseminars der Bundeskonferenz der Kommunalarchive (BKK) in Eisenach vom 23. - 25. November 2011. Münster 2012 (Texte und Untersuchungen zur Archivpflege 25), S. 149-161; Thomas NotTHOFF, Urheberrecht und Archivbenutzung am Beispiel der Examensarbeiten der Ersten Staatsprüfung für Lehrämter an Schulen im Landesarchiv NRW. Transferarbeit im Rahmen des Vorbereitungsdienstes zum höheren Archivdienst Landesarchiv NRW Abt. Westfalen. Archivschule Marburg, 44. WK. [Marburg] 2011, abrufbar unter: http://www.archive.nrw.de/lav/abteilungen/f achbereich_grundsaetze/BilderKartenLogosDateien/Transferarbeiten/Notthoff_Transferarb eit.pdf, zuletzt abgerufen am 22.05.2018.

RuZ 2. Jg. 3/2021 
2.) Die Hürden für die Zuerkennung des Urheberrechtsschutzes sind in der Rechtsprechung immer weiter abgesenkt worden („kleine Münze“) - das Problem betrifft eben mitnichten „nur“ Fotos, Filme oder Zeitungsartikel, sondern kann theoretisch eine große Menge an Alltagsschriftgut aus „normalen“ amtlichen Sachakten umfassen.

3.) Damit zusammenhängend ist bei vielen urheberrechtlich geschützten Werken in Archivgut völlig unklar, wer überhaupt der oder die Urheber sind. Die bisherigen Regelungen zu den „verwaisten Werken“ im UrhG ( $\mathbb{6} 61 \mathrm{ff}$.) sind für Archive weitgehend untauglich.

4.) Ebenso ungeklärt beziehungsweise umstritten ist, inwiefern bei bestimmten urheberrechtlich geschützten Werken im Sinne des konkludenten Handelns beziehungsweise der Zweckübertragungslehre Nutzungsrechte - und wenn ja, in welchem Umfang - auf das Archiv übergehen (These: Wer etwas im Archiv direkt oder bei einer anbietungspflichtigen Stelle abgibt beziehungsweise für letztere tätig ist, überträgt damit stillschweigend und mittelbar alle notwendigen Nutzungsrechte an das Archiv). Dies ist sogar für in Arbeits- und Dienstverhältnissen entstandene Schriftstücke nicht unumstritten ${ }^{28}$, erst recht stellt sich die Problematik - insbesondere wegen $₫ 44$ Abs. 1 UrhG - bei privatem Schriftgut (Schenkungen, Deposita).

5.) Eine parallele ungelöste Frage ist, ob unikales Archivgut als veröffentlicht anzusehen ist oder nicht ${ }^{29}$. Damit verbunden auch die Frage, ob schon die Zugänglichmachung im Lesesaal - und nicht erst im Internet - als Veröffentlichung einzustufen ist.

6.) Aus all dem ergibt sich ein nahezu unlösbarer Zielkonflikt für Archive: Eine konsequente Beachtung des Urheberrechts führt nämlich zur Ungleichbehandlung von nach Archivgesetz gleich zu behandelnden Unterlagen und deren Benutzern, ohne dass es dafür im Archivgesetz selbst eine hinreichend transparente Grundlage gäbe. Eine solche Unterordnung der Benutzung unter Rechtsvorschriften außerhalb der Archivgesetze ist den Benutzern kaum zu vermitteln ${ }^{30}$.

28 Vgl. Stephan DusiL, Zwischen Benutzung und Nutzungssperre. Zum urheberrechtlichen Schutz von archivierten Fotografien, in: Archivar 61/2 (2008), S. 124-132; kritisch gegenüber der Übertragung selbst bei in Arbeits- und Dienstverhältnissen entstandenem Schriftgut ( 443 UrhG) [KLA], Die bisherigen Reformen des Urheberrechts (wie Anm. 27), S. 28f. Der Verfasser teilt diese Bedenken nach Konsultation des einschlägigen Kommentars von Thomas DreiER / Gernot Schulze, Urheberrechtsgesetz. Verwertungsgesellschaftengesetz. Kunsturhebergesetz. Kommentar. München ${ }^{6}$ 2018, hier S. 927-930 (\$43 UrhG, Einräumung von Nutzungsrechten) allerdings nicht.

29 Die Neuregelungen in den $\iint$ 60c , e und f UrhG von 2018 helfen in dieser Frage aus Sicht des Verfassers nur bedingt, weil die weiterhin bestehenden Einschränkungen kaum alltagspraktisch umsetzbar sind.

30 Vgl. dazu, wenngleich am Beispiel des KunstUrhG aber dennoch vergleichbar RosenPLÄNTER, Menschenleere Strände (wie Anm. 26), 125f. Im Übrigen ist dieser Fall nicht mit demjenigen von außerhalb der Archivgesetze festgelegten Einsichtsrechten - etwa nach dem Umweltinformationsgesetz - vergleichbar, weil diese eine Besser- und keine Schlechterstellung der Benutzer bedeuten, vgl. dazu insgesamt Christian ReInHardt, Die wissenschaftliche 
Leider bietet die jüngere Rechtsprechung zu dieser Thematik wenig Erbauliches für Archive. Ich konzentriere mich im Folgenden nur auf drei Aspekte:

1.) Die konkludente Einwilligung beziehungsweise Zweckübertragungslehre sowie damit zusammenhängend die Frage der Erstveröffentlichung,

2.) die Erstellung von Reproduktionen,

3.) das generelle Verhältnis von Archivrecht und Urheberrecht.

$\mathrm{Zu}$ 1.)

Bereits 2006 hatte das Landgericht Berlin verneint, dass an einen Bundesminister (!) gerichtete und später ins Bundesarchiv gelangte Briefe als veröffentlicht anzusehen seien, denn die Briefe seien nicht vom Urheber selbst im Archiv abgegeben worden. Die Anbietungspflicht und das archivische Benutzungsrecht spielten für das Landgericht keine Rolle, ergo fand aus dieser Sicht weder eine Veröffentlichung und also erst recht keine Übertragung von Nutzungsrechten statt ${ }^{31}$. Weil das Werk nicht veröffentlicht sei, greife auch das Zitatrecht $(\mathbb{5} 5 \mathrm{UrhG})$ nicht - zwar könne die Abwägung zwischen Presse- und Meinungsfreiheit gegenüber Persönlichkeits- und Eigentumsrecht zu einer Einschränkung des Erstveröffentlichungsrechts führen, doch im vorliegenden Fall wäre als schonendste Variante maximal das auszugsweise Zitieren zulässig gewesen, keinesfalls aber eine vollständige Veröffentlichung.

Ist nun allerdings - jenseits der Frage der Veröffentlichung in einem anderen Medium - schon die Vorlage im Lesesaal eine solche „Veröffentlichung“? Diese Frage ist zuletzt unterschiedlich beantwortet worden: Das Verwaltungsgericht Magdeburg verneinte dies im Hinblick auf den Zugang zu urheberrechtlich geschützten Unterlagen nach Informationsfreiheitsgesetz: dies sei keine Veröffentlichung - gleiches kann parallel für die Benutzung im Lesesaal konstatiert werden ${ }^{32}$. Allerdings ging es in diesem Fall um Unterlagen, die im Auftrag der Behörde entstanden sind. Ganz anders sieht dies bei im Verwaltungsverfahren von Dritten eingereichten Unterlagen aus: Hier hat das Bundesverwaltungsgericht zuletzt in einem Verfahren nach Umweltinformationsgesetz entschieden, dass in diesem Fall von einer konkludenten Zustimmung zur Zugänglichmachung nicht ausgegangen werden könne, denn die Unterlagen seien nur für

Nutzung von Archivgut mit Sozial- und Steuerdaten, in: Archivar 66/4 (2013), S. 439-443. $\mathrm{Ob}$ die Nutzungsversagungsgründe der Archivgesetze eine Anwendung des Urheberrechts implizieren könnten, erscheint doch eher zweifelhaft.

31 LG Berlin, Beschl. v. 10.10.2006 - 16 O 908/06; im Verfahren ging es um Briefe von Günter Grass und eine Veröffentlichung durch die F.A.Z.; zu diesem Fall insgesamt Andrea HäNGER, Urheberrecht im Archiv: das Beispiel des Bundesarchivs, in: Paul Klimpel (Hg.), Mit gutem Recht erinnern. Gedanken zur Änderung der rechtlichen Rahmenbedingungen des kulturellen Erbes in der digitalen Welt. Hamburg 2018 (DOI: https://doi.org/10.15460/HUP .178), S. 25-35.

32 VG Magdeburg, Urt. v. 23.01.2018 - 6 A 343/16 MD; vgl. dazu auch Klaus Graf, Verwaltungsgericht Magdeburg: Urheberrecht kein Ausschlussgrund für IFG-Auskünfte (Blogbeitrag vom 20.02.2018, abrufbar unter: https://archivalia.hypotheses.org/70584, zuletzt abgerufen am 14.06.2020). 
die Behörde und niemand anderen bestimmt gewesen - und dass nur ein Antragsteller Einsicht begehre, ändere nichts, denn der Personenkreis sei laut Gesetz grundsätzlich unbegrenz $t^{33}$. Streng genommen wird damit - und entgegen dem Verwaltungsgericht Magdeburg - eine Veröffentlichung durch Zugänglichmachung für alle Unterlagen, egal ob in amtlichem Auftrage entstanden oder nicht, konstatiert. Auch wenn beide Verfahren das Informationsrecht betrafen, sind sie nach Ansicht des Verfassers analog auf die archivische Benutzung anwendbar (Jedermann-Recht der meisten Archivgesetze et cetera). Unter dieser Maßgabe dürfte eine erhebliche Menge von Unterlagen egal ob amtlicher oder nicht-amtlicher Provenienz! - selbst im Lesesaal nicht mehr vorgelegt (und erst recht nicht reproduziert oder veröffentlicht) werden, sofern sich darin urheberrechtlich geschützte Unterlagen Dritter befinden ${ }^{34}$ - und wegen der oben genannten geringen Schutzhöhe kann darunter ziemlich viel fallen. Zwar lässt das Bundesverwaltungsgericht insofern einen Spalt offen, als dass eine Zugänglichmachung bei überwiegendem öffentlichen Interesse zulässig wäre ${ }^{35}$. Aber auch das liefe wiederum auf eine Einzelfallprüfung hinaus, die in der archivischen Alltagspraxis nicht zu leisten und überdies mit neuen rechtlichen Unsicherheiten verbunden ist.

Alles in allem muss festgehalten werden, dass die - an sich gute und klare - Idee einer konkludenten Zustimmung und Zweckübertragung von Nutzungsrechten an das Archiv im Hinblick auf die archivgesetzliche Anbietungspflicht jedenfalls aktuell bedauerlicherweise nicht durch die Rechtsprechung gedeckt ist: Für urheberrechtlich geschütztes Schriftgut Dritter auch in amtlichen Unterlagen werden keine Nutzungsrechte stillschweigend durch Abgabe an das Archiv übertragen, erst recht gilt dies für entsprechende Unterlagen in nicht-amtlichen Unterlagen ${ }^{36}$. In Übereinstimmung damit findet eben auch keine stillschweigende Zustimmung zur Veröffentlichung dadurch statt, dass der Urheber mit der Anbietung, Übernahme und Zugänglichmachung nach Archivgesetz rechnen musste: solches Archivgut ist nicht im Sinne des Urheberrechts veröffentlicht. Selbst die Vorlage im Lesesaal könnte man je nach Auslegung als unzulässig betrachten. Die von den Gerichten theoretisch zugelassene Abwägung zugunsten des Informationsinteresses läuft tendenziell auf eine praktisch bei der Masse nicht durchführbare Einzelfallprüfung hinaus ${ }^{37}$.

33 BVerwG, Urt. v. 26.09.2019 - 7 C 1/18.

34 Beispiele bei [KLA], Die bisherigen Reformen des Urheberrechts (wie Anm. 27), S. 29.

35 Im vorliegenden Fall ging es jedoch um kommerzielle Interessen des Antragstellers.

36 Diese Problematik auch schon erörtert etwa bei Polley, Aspekte des Urheberrechts (wie Anm. 27), S. 140-144.

37 Insofern hilft weder der Umstand, dass überwiegend das Schriftgut Dritter in amtlichen Unterlagen nicht die Schöpfungshöhe erreichen wird, noch die Lösung über die Qualifizierung urheberrechtlich geschützter Unterlagen als unwesentliches Beiwerk ( $\$ 57$ UrhG) (vgl. [KLA], Die bisherigen Reformen des Urheberrechts [wie Anm. 27], S. 30f.) - zumal diese Möglichkeit ohnehin immer weiter eingeschränkt wird, vgl. DreIER / SCHUlze, Urheberrechtsgesetz (wie Anm. 28), S. 1103-1105 (\$57) -, weil dies ebenfalls eine proaktive Prüfung erfordert. 
Gesetzt den Fall, man geht mit der herrschenden Meinung zumindest von der Zulässigkeit der Vorlage urheberrechtlich geschützten Archivgutes im Lesesaal aus, so stellt sich die Frage, ob Reproduktionen wenigstens zum eigenen Gebrauch erstellt werden können $^{38}$ - etwas, das wohlgemerkt sogar für personenbezogenen Schutzfristen unterliegendes Archivgut gang und gäbe ist. Eine solche Reproduktion kann theoretisch auf $\$ 53$ Abs. 1 Satz 2 UrhG gestützt werden, indem angenommen wird, das Archiv agiere quasi nur wie ein Kopiergerät oder privater Werkunternehmer für den Besteller (= Benutzer). Doch auch hier sieht es nach einer Entscheidung des Oberverwaltungsgerichts NRW von 2016 zum Informationsfreiheitsgesetz mittlerweile schlecht aus ${ }^{39}$ : Denn demnach ist die öffentlich-rechtliche Beziehung zwischen Behörde und Antragsteller (sc. im Archiv: Benutzer) anders geregelt als privatrechtliche Werkverträge, weil eine differenzierte Antragsprüfung durch die Behörde stattfindet und diese an Gesetze gebunden ist - durch die Übersendung des Werkes zum dauerhaften eigenen Gebrauch würde die Behörde aus ihrer behördlichen Sphäre heraustreten, dafür gebe es keine Grundlage.

Im Klartext: Von Archivalien, die keinen urheberrechtlichen Schutz genießen, können - sogar unter noch laufenden Schutzfristen - Reproduktionen zum eigenen Gebrauch für Benutzer erstellt werden, bei urheberrechtlich geschütztem Archivgut kann dies sogar dann noch unzulässig sein, wenn die archivrechtlichen Schutzfristen schon abgelaufen sind. Dass diese Ungleichbehandlung absurd wirkt und den Benutzern kaum logisch erklärbar ist, darüber braucht sich jedoch kein Archiv zu wundern.

Angesichts der geschilderten Verhältnisse könnte man allerdings zu der Überlegung kommen: Wieso sollte sich ein öffentliches Archiv überhaupt für das Urheberrecht interessieren? Denn in den Archivgesetzen wird das Urheberrecht üblicherweise nicht erwähnt ${ }^{40}$ - im Gegensatz etwa zu Bibliotheks- oder Informationsfreiheitsgesetzen ${ }^{41}$. Der Fall ist auch nicht mit demjenigen der Anwendung des BGB bei Schenkungen

38 In der Praxis der öffentlichen Archive wird dies wohl überwiegend für unzulässig gehalten, wie indirekt der Umstand zeigt, dass im Falle der Selbstherstellung von Reproduktionen urheberrechtlich geschützte Archivalien vielfach von dieser Erlaubnis ausgenommen sind (ob diese Einschränkung auch für Benutzer selbst angesichts der Schrankenregelung zur Privatkopie in $\$ 53$ UrhG zulässig ist, soll hier nicht diskutiert werden). Befürwortend dagegen Klaus GrAF, Fragwürdiges Urteil des VG Braunschweig (Blogbeitrag vom 16.09.2008, abrufbar unter: https://archivalia.hypotheses.org/24280, zuletzt abgerufen am 22.04.2020).

39 OVG Nordrhein-Westfalen, Urt. v. 24.05.2016 - 15 A 2051/14. Dem Verfahren lag das Begehren auf Erstellung und Übersendung einer Kopie eines Pornofilms durch die Bundesprüfstelle für jugendgefährdende Medien zugrunde.

40 Auf Sonderfälle wie $\int 9$ Abs. 8 ArchGB (Geltung des KunstUrhG bei im Landesarchiv verwahrten audiovisuellen Werken) wird hier nicht eingegangen.

41 Vgl. etwa $\$ 16 a \mathrm{DNBG}$; 6 IFG.

RuZ 2. Jg. 3/2021 
oder Deposita vergleichbar, weil hier die „Anbietung“ und Übergabe nicht auf öffentlich-rechtlichen, sondern privatrechtlichen Grundlagen beruht. Für die Benutzung trifft dies jedoch nicht zu, hier sind die archivgesetzlichen Regelungen abschließend. Allerdings wird das Urheberrecht sehr wohl in einigen Archivbenutzungsordnungen erwähnt. Wichtiger noch ist allerdings, dass das Urheberrecht nach Art. 73 GG Abs. 1 Nr. 9 der ausschließlichen Gesetzgebungszuständigkeit des Bundes unterfällt, weshalb es gemäß Art. 31 GG den Vorrang genießt.

Diese zweite Auslegung eines absoluten Vorrangs des Urheberrechts ist spätestens mit dem Urteil des Gerichtshofs der Europäischen Union zum „Fall Volker Beck“ vom Juli 2019 als definitiv gültig anzusehen ${ }^{42}$ : Demnach gibt es außerhalb der Schrankenregelungen des UrhG keine Eingriffsmöglichkeiten in die Rechte des Urhebers sofern in dessen Rechte eingegriffen wird, vermögen dies weder das Archivrecht noch Grundrechte wie Informations- und Pressefreiheit als solche zu rechtfertigen. Zwar verneinte der EuGH die schrankenlose Gültigkeit des Urheberrechts, es sei immer eine Rechtsgüterabwägung bei konkurrierenden Grundrechten notwendig - aus den schon oben genannten Gründen ist dies aber für die archivische Praxis wenig hilfreich. Indirekt wurden die Möglichkeiten der Archivbenutzung zudem noch in einem anderen Punkt weiter verschlechtert: Das Zitatrecht nach $\$ 51$ UrhG greift demnach nur, wenn das zugrunde liegende Werk veröffentlicht ist - kombiniert mit den weiter oben erwähnten Entscheidungen, dass eine Abgabe ins Archiv keine Veröffentlichung darstellt, schränkt dies die Möglichkeiten der Benutzer weiter ein ${ }^{43}$. Die Auslegung des EuGH ist insofern sogar noch restriktiver als die oben angeführte des Landgerichts Berlin, in welcher zumindest die Möglichkeit der Abwägung auch für unveröffentlichtes Material als denkbar angesehen wurde.

Letztlich setzen die Gerichte mit der Bevorzugung der Position der Urheber nur um, was die Gesetzgeber in ihren Gesetzesformulierungen immer wieder zum Ausdruck gebracht haben. Aus diesem Grund dürften auf mittlere Sicht aber auch Hoffnungen auf eine - zweifellos sinnvolle - allgemeine Verjährungsfrist (Kulturgüterschranke) weiterhin zwecklos sein ${ }^{44}$. Angesichts dessen müssen Archive sich also auch weiterhin fragen, wie sie mit diesem Problem - das Stand 2020 alle Archivalien nach der Mitte des 19. Jahrhunderts betrifft - praktisch umgehen wollen. Aus Sicht des

42 EuGH, Urt. v. 29.07.2019 - C-516/17. Zum dahinter stehenden Fall vgl. Martin Gerecke, Der Fall Volker Beck vor dem EuGH. Eine verquere Sexualmoral und das Zitatrecht (Blogbeitrag vom 29.06.2020, abrufbar unter: https://www.lto.de/recht/hintergruende/h/eugh-urt eil-c51617-volker-beck-urheberrecht-zitat-spiegel-online, zuletzt abgerufen am 04.08.2019). Diese Linie wurde auch in einem anderen Urteil vom gleichen Tag zu den sogenannten „Afghanistan-Papieren“ bestätigt: EuGH, Urt. v. 29.07.2019 - C-469/27.

43 Knappe Kritik dazu aus Archivsicht bei Klaus Graf, Kein Zitieren von Unveröffentlichtem? (Blogbeitrag vom 31.07.2019, abrufbar unter: https://archivalia.hypotheses.org/101540, zuletzt abgerufen am 21.06.2020).

44 Vgl. Oliver Hinte, Schutzfristen und kulturelles Erbe: Nach 25 Jahren ist Schluss (Blogbeitrag vom 19.01.2018, abrufbar unter: https://irights.info/artikel/schutzfristen-und-kulturelle s-erbe-nach-25-jahren-ist-schluss/28897, zuletzt abgerufen am 27.02.2018). 
Verfassers gibt es vier Strategien, von denen zwei nicht wirklich seriös sind, aber die Extreme des Problems aufzeigen. Wirklich befriedigend ist allerdings keine davon:

1.) Konsequente Verweigerung jeder Benutzung für alle Unterlagen ab einem Stichjahr X minus 170 Jahre (maximale Lebensdauer zuzüglich 70 Jahre Urheberrechtsschutz nach Tod): Damit ist man urheberrechtlich definitiv auf der sicheren Seite, verstößt aber zugleich gegen alle Regeln der archivarischen Berufsethik und in vielen Fällen paradoxerweise sogar gegen das Urheberrecht selbst: denn wegen fehlender Einzelfallprüfung wird der Ablauf des urheberrechtlichen Schutzes nicht bemerkt und die Benutzung unzulässigerweise verweigert werden.

2.) Ignorieren des Urheberrechts: Dies bedeutet, in vollem Bewusstsein das Risiko einer zivilrechtlichen Inanspruchnahme wegen Urheberrechtsverletzungen nach den $\$ \mathbb{\$}$ 97-105 UrhG, theoretisch auch der strafrechtlichen Sanktionen nach den $\mathbb{\int} \mathbb{S} 106-111 \mathrm{a}$ UrhG ${ }^{45}$ einzugehen. Selbst wenn diese Bereitschaft vorhanden ist, muss einerseits immer damit gerechnet werden, dass urheberrechtliche Fragen von externer Seite (Benutzer, Fachaufsicht) an das Archiv herangetragen werden. Zum anderen ist es mit der berufsständischen Kollegialität nicht vereinbar, den Nachfolgern einen Sack ungeklärter Rechtsprobleme zu hinterlassen.

3.) Einzelfallprüfung: Diese lohnt sich bei klar identifizierbaren und herausragenden Stücken beziehungsweise Archivguttypen, auch bei klar einem Urheber zuzuordnenden Konvoluten. Hier kann auch ein Nacherwerb von Nutzungsrechten versucht werden. Selbst dann sind allerdings erhebliche Probleme zu erwarten ${ }^{46}$. Für die Masse der verstreut in den Akten vorkommenden urheberrechtlich geschützten Unterlagen ist sie undurchführbar.

4.) Risikomanagement ${ }^{47}$ : Um der gerichtlich zugelassenen Rechtsgüterabwägung (Art. 14 vs. Art. 5 Abs. 1 und 3 GG et cetera) ebenso gerecht zu werden wie dem Erfordernis praktischer Durchführbarkeit kann eine Risikoanalyse durchgeführt werden $^{48}$. Ziel ist es, massenhaft bestimmte Gruppen von Archivalien typisierend ohne Einzelfallprüfung zu behandeln. Zu berücksichtigen wären wesentlich Eintrittswahrscheinlichkeit einer Klage/Beschwerde multipliziert mit dem Schadenspotenzial. So ist zum Beispiel bei - für Archiven typischen - Unikaten wegen der fehlenden Veröffentlichung und dem fehlenden kommerziellen Zweck ein Scha-

45 Hier dürfte es sich allerdings tatsächlich nur um ein theoretisches Risiko handeln: Da die Verletzung eben nicht zu privaten Zwecken stattfindet und man sich in der Praxis immer auf Unwissenheit zurückziehen kann, ist eine Verfahrenseinstellung nach den $\$ \mathbb{S} 153$ oder 172 Abs. 2 StPO der ganz überwiegend wahrscheinliche Ausgang.

46 Vgl. das Beispiel bei KrÄMER / ZiLles, Urheberrecht als archivische Herausforderung (wie Anm. 27), bes. S. 112-118.

47 Vgl. zu diesem Thema paradigmatisch Paul KLimpel, Kulturelles Erbe digital. Eine kleine Rechtsfibel. Berlin 2020 (DOI: https://doi.org/10.12752/2.0.004.0), hier S. 89f., sowie insgesamt die Hinweise auf https://www.digis-berlin.de, zuletzt abgerufen am 02.05.2021.

48 Hinweise dazu gibt [KLA], Die bisherigen Reformen des Urheberrechts (wie Anm. 27), S. 24-35; vgl. auch Paul Klimpel / Fabian Rack / John H. Weitzmann, Neue rechtliche Rahmenbedingungen für Digitalisierungsprojekte von Gedächtnisinstitutionen. 4., gänzlich neu bearbeitete Auflage. November 2017 (DOI: https://doi.org/10.12752/2.0.002.3), S. $10 \mathrm{f}$. 
densersatzanspruch kaum zu begründen: Es gibt keinen „freien Markt“ für solche Stücke. Bei verwaisten Werken haben etwaige Kläger schon Probleme, ihren Urheberrechtsanspruch nachzuweisen (fehlende „Aktivlegitimation“). Weiter kann man sich der Auslegung anschließen, dass für die ganz überwiegende Masse des infolge der Anbietungspflicht in das öffentliche Archiv gelangten amtlichen Registraturgutes, auch soweit es Schöpfungshöhe nach $\$ 2$ Abs. 2 UrhG aufweist, gemäß den $\$ \mathbb{S} 43,69 \mathrm{~b}$ (Arbeits- und Dienstverhältnisse) die Nutzungsrechte an das Archiv übergehen ${ }^{49}$. Alltagsschriftgut, wenn auch mit Schöpfungshöhe, in amtlichen Sachakten wird dann eben anders behandelt als Fotos eines berühmten Pressefotografen in einem Nachlass. Eine weitere Differenzierung ist diejenige nach Benutzungsarten: von der Benutzung im Lesesaal über die Erstellung von Reproduktionen bis zur Präsentation von freien Digitalisaten im Internet. Faktisch wird sich die Differenzierung der zugelassenen Benutzungsarten wiederum am Typus des Archivgutes orientieren. Liegt eine entsprechende Typisierung vor, kann eine aufwendige Einzelfallprüfung nach 3.) auf bestimmte Einzelstücke, Konvolute oder besondere Benutzungszwecke begrenzt werden. Letztlich bedeutet diese Lösung allerdings auch, im Sinne der Benutzbarkeit von Archivgut den urheberrechtlichen Schutz für bestimmte Gruppen von Archivalien (wie etwa amtliche Unterlagen) geringer zu gewichten als für andere (wie etwa private Nachlässe), obschon dies natürlich im Urheberrecht selbst keinerlei Stütze findet.

Vermutlich werden die meisten Archive nach den Strategie Nr.3.) und 4.) verfahren und sich irgendwo in der Grauzone zwischen den Extremen 1.) und 2.) bewegen. Da "völlig" rechtskonformes Handeln unter den gegebenen Bedingungen bis auf weiteres kaum möglich ist, sollte man zumindest die eigene Vorgehensweise reflektieren und dokumentieren. Ob künftig die angestrebte Novellierung des UrhG im Zuge der Umsetzung der „DSM-Richtlinie“ $50 \mathrm{zu}$ wesentlichen Verbesserungen, etwa durch die Möglichkeit des Erwerbs kollektiver Lizenzen, führt, bleibt abzuwarten.

\section{Erschließung}

In einem Prozess um Zugang zu Unterlagen des Bundesamtes für Verfassungsschutz über NS-Verbrecher (Eichmann, Alois Brunner) beschäftigte sich das Bundesverwaltungsgericht zuletzt im Dezember 2019 mit Fragen der Schriftgutverwaltung und damit zusammenhängend der Vergabe von Schutzfristen ${ }^{51}$. Während in der medialen Berichterstattung vor allem die Äußerungen des damaligen BfV-Präsidenten Maaßen,

49 Wie weiter oben erwähnt, ist dies strittig.

50 Richtlinie 2019/790-EU über das Urheberrecht und die verwandten Schutzrechte im digitalen Binnenmarkt und zur Änderung der Richtlinien 96/9/EG und 2001/29/EG.

51 BVerwG, Urt. v. 11.12.2019 - 6 C 21/18. 
im Falle des Unterliegens vor Gericht das Bundesarchivgesetz ändern zu wollen, für Aufsehen sorgten ${ }^{52}$, sind aus archivischer Sicht vor allem folgende Punkte relevant:

1.) „Unterlagen“ können auch einzelne Schriftstücke in Akten sein.

2.) „Entstehung“ bezieht sich auf abgeschlossene Vorgänge innerhalb von Akten und damit auf einzelne Schriftstücke innerhalb derselben -, nicht auf die Gesamtakte.

3.) Die allgemeine dreißigjährige Schutzfrist ist daher jeweils gesondert für Vorgänge innerhalb von Akten zu bilden - deren letzte substanzielle Bearbeitung ist mit der z.d.A.-Verfügung abgeschlossen, eine spätere Wiederaufnahme führt zu einem neuen Vorgang.

Unbeschadet des Umstandes, dass hier die speziellen Formulierungen des Bundesarchivgesetzes zugrunde liegen - wobei andere Archivgesetze aber durchaus ähnliches aufweisen ${ }^{53}$ - klingt das in der Theorie erst einmal wie eine Horrorvision: allgemeine Schutzfristen wären demnach für Sachakten ${ }^{54}$ nicht einheitlich, sondern für jeden Vorgang separat festzusetzen. Wiederum kollidiert die Auslegung durch das Gericht also mit dem archivischen Erschließungsalltag, wo wohl nur selten in dieser Form verfahren werden dürfte. In der Praxis jedoch stärkt das Gericht damit die Möglichkeiten der Benutzung und Zugänglichmachung: Teilakten können nun, sofern praktikabel, schneller und unkomplizierter für die Benutzung freigegeben werden, während umgekehrt Benutzer kaum je von schon abgelaufenen Schutzfristen für Vorgänge erfahren werden, solange sie keinen Zugang zur Gesamtakte haben. Freilich sind Archive insofern aufgefordert, hier benutzungsfreundlich zu verfahren.

Gerichtlich bestätigt wird damit aber auch, dass Schutzfristen sich stets nur nach der letzten substanziellen Bearbeitung zu richten haben, während spätere flüchtige Vermerke oder Verfügungen keinen Einfluss auf die Schutzfristenvergabe haben. Im Hintergrund dieser Entscheidung steht das - wiederum für Archive förderliche Ziel des Bundesverwaltungsgerichts, eine Laufzeitverlängerung von Unterlagen durch regelmäßig wiederholte substanzlose Bearbeitungen und damit Unterlaufen der Anbietungspflicht ad infinitum zu verhindern.

52 Vgl. zum Prozess etwa https://www.rnd.de/politik/urteil-verfassungsschutz-muss-einsicht -in-akte-von-ns-tater-geben-W2XBBNY2PF5N2GYFXO2KSVMRTI.html, zuletzt abgerufen am 25.02.2020 und https://www.welt.de/geschichte/article204255816/Geheimhaltung-W as-man-ueber-den-NS-Taeter-Alois-Brunner-noch-wissen-will.html, zuletzt abgerufen am 25.02.2020. Dass der vormalige BfV-Präsident Maaßen die Zurückweisung der Revision des BfV gar nicht mehr im Amt erlebte, gehört zu den kuriosen Randaspekten des Verfahrens.

53 Vgl. etwa die Begriffsbestimmungen für "Schriftgut" und „Archivgut" in $\ 2$ Abs. 1 und 2 NArchG; ähnlich $\$ 2$ Abs. 1 ArchivG NRW.

54 Für personenbezogene Akten stellt das BVerwG durchaus auf die Gesamtakte ab, vgl. dazu auch die schon zitierte Entscheidung von BVerwG, Urt. v. 30.01.2019 - 6 A 1/17. 


\section{IV. Überlieferungsbildung}

Die Dauerfehde „Presse vs. Geheimdienste“ sorgt auch in anderen Arbeitsfeldern für aufschlussreiche Auslegungen ${ }^{55}$. Weniger erbaulich ist dabei die in mehreren Entscheidungen vom Bundesverwaltungsgericht - wiederum mit Bezug auf das BArchG, aber pars pro toto auf alle Archivgesetze anwendbar - festgestellte gänzlich fehlende Sanktionsmöglichkeit bei Nichterfüllung der Anbietungspflicht ${ }^{56}$. Dass das Bundesverwaltungsgericht diesbezüglich die in der archivrechtlichen Diskussion immer mal wieder angeführten Straftatbestände ( $\mathbb{S} 133,274$ StGB) nicht einmal als Möglichkeit erwähnt, bestätigt indirekt nur die auch in der jüngeren Literatur getroffene Feststellung, dass von dieser Seite aus keinerlei Hilfe zu erwarten ist ${ }^{57}$ : Wegen Aktenvernichtungen ohne vorherige Anbietung an das Archiv wurden bisher und werden wohl auch künftig keine Behördenmitarbeiter hinter Schloss und Riegel einquartiert.

Günstiger ist indes eine weitere Teilentscheidung des Bundesverwaltungsgerichts aus dem schon im vorigen Abschnitt erwähnten Urteil zum Zugang zu Unterlagen des $\mathrm{BfV}^{58}$. In der aktuellen Diskussion sind die Anwendbarkeit der Theorie vom „Löschungssurrogat", das Vordringen von die Anbietungspflicht aushöhlenden datenschutzrechtlichen Regelungen sowie die Erschwernisse für Archive durch (bundes-)gesetzliche Löschungs- und Vernichtungsgebote virulent ${ }^{59}$. Einigermaßen nachteilig für

55 Vgl. etwa die Aufbereitung verschiedener Entscheidungen bei PARTSCH, Bundesarchivgesetz. Handkommentar (wie Anm. 17), hier S. 140 Rn. 22 und ff. (zu \$6 BArchG) und S. 209 Rn. 36 mit Anm. 102.

56 Vgl. etwa BVerwG, Beschl. v. 12.09.2017 - 6 A 1/15; ebenfalls erwähnt in der schon im vorigen Abschnitt besprochenen Entscheidung BVerwG, Urt. v. 30.01.2019 - 6 A 1/17. Hintergrund ist wiederum die Möglichkeit der Benutzung von Unterlagen in der Behörde nach BArchG, die eine Anreizfunktion für die Abgabe ausüben soll. Die fehlende Sanktionsmöglichkeit wurde zuletzt gar explizit im aktuell wohl bekanntesten archivrechtlichen Streitfall, nämlich dem Umgang mit in private Hände gelangten Unterlagen politischer Amtsträger, angeführt: BVerfG, Beschl. v. 20.06.2017 - 1 BvR 1978/13.

57 Vgl. zuletzt Jost Hausmann, Sicherung im Vorfeld. Aktenvernichtung und -entfremdung als Rechtsfrage, in: BeCKeR u.a. (Hg.), Nicht nur Archivgesetze (wie Anm. 1), S. 57-84, hier 6171 (die potenzielle Strafbarkeit zumindest nach $\$ 133$ StGB bejahend, allerdings ohne praktische Folgen); eindeutig in dieser Richtung bereits Herbert GÜNTHER, Archive und Verwaltung oder: Über die Grenzen des Archivrechts, in: Irmgard Christa BECKER / Dominik HAFFER / Volker Hirsch / Karsten Uhde (Hg.), Archiv - Recht - Geschichte. FS Rainer Polley. Marburg 2014 (Veröffentlichungen der Archivschule Marburg 59), S. 195-242, hier S. $227-$ 235 (klare Ablehnung einer möglichen Strafbarkeit).

58 BVerwG, Urt. v. 11.12.2019 - 6 C 21/18, hier Rn. 12, 45-48.

59 Vgl. Clemens Rehm, Löschkultur versus Anbietungspflicht. Standortbestimmung und Perspektiven, in: Becker / U.A., Nicht nur Archivgesetze (wie Anm. 1), S. 85-117, bes. 90-107; Gregor PATT, Chancen oder Stolperfallen? Rechtliche Vorgaben zur Überlieferungsbildung außerhalb des Archivgesetzes, in: Archive im Rechtsstaat (wie Anm. 1), S. 71-80, hier S. 7880; Michael ScHolz, „... wäre es nicht gerechtfertigt, der Überlieferung von Unterlagen absoluten Vorrang ... einzuräumen." Ausnahmen von der Anbietungspflicht als Problem der Überlieferungsbildung, in: Archivpflege in Westfalen-Lippe 83 (2015), S. 37-43; Arnd VolLMER, Das Verhältnis von bundesrechtlichen Löschungsgeboten und landesrechtlicher Anbietungspflicht. Diskussionsgrundlage [2014], abrufbar unter: http://www.bundesarchiv.de/DE /Content/Downloads/KLA/verhaeltnis-loeschungsgebote-anbietungspflicht.pdf?_blob=pu 
Archive ist in diesem Zusammenhang bekanntlich der seit 2017 geltende $\$ 6$ Abs. 2 Nr. 2 BArchG, der für bestimmte Unterlagen, die gesetzlichen Löschungs- und Vernichtungsgeboten unterliegen, die Gültigkeit der Anbietungspflicht aushebelt und mittelbar auch für Landes- und Kommunalarchive Folgen haben könnte ${ }^{60}$. Das Gericht dreht nun aber den üblichen archivischen Ansatz - die Archivierung ist ein Surrogat für die Löschung oder gegebenenfalls auch Vernichtung - einfach um: Die Anbietung und Übernahme von Unterlagen sei gerade kein Surrogat für die Aktenvernichtung, sondern umgekehrt stehe prinzipiell jede Aktenvernichtung erst einmal unter dem Vorbehalt der Anbietung und Übernahme. Zwar bezieht sich das Bundesverwaltungsgericht dabei auf $\mathbb{1 3}$ Abs. 3 Satz 7 BVerfschG, der eine Anbietung an das Bundesarchiv vor der Vernichtung vorschreibt. Daraus kann aber nicht gefolgert werden, ohne diesen Passus wäre eine Anbietung nicht erforderlich oder zulässig, denn dieser Passus zur Anbietungspflicht im BVerfschG ist wiederum nur wegen der Ausschlussregelung des $\$ 6$ Abs. 2 Nr. 2 BArchG nötig. Ansonsten geht jedoch nach dieser Entscheidung prinzipiell die archivgesetzliche Anbietungspflicht jeder spezialgesetzlichen Löschungs- und Vernichtungsregelung vor ${ }^{61}$.

Obwohl es sich innerhalb des Urteils nur um einen Nebenaspekt handelt ${ }^{62}$ und der Einzelfall auf das BfV bezogen ist, hat das Bundesverwaltungsgericht damit mutmaßlich unintendiert - den Archiven indirekt ein mächtiges Argument an die Hand gegeben: Denn künftig kann bei verweigerter Anbietung oder Abgabe unter Berufung auf - auch bundesgesetzliche! - Löschungs- und Vernichtungsregelungen ${ }^{63}$ stets der Vorrang der Anbietungspflicht als höchstgerichtliche Festlegung entgegenge-

blicationFile, zuletzt abgerufen am 05.01.2018, hier bes. S. 4ff. (mit Betonung des Vorrangs der landesrechtlichen Anbietungspflicht auch bei bundesrechtlichen Löschungsgeboten, soweit die entsprechenden Bundesgesetze die Anbietung nicht ausdrücklich verbieten).

60 „Von der Anbietungspflicht ausgenommen sind [...] Unterlagen, die nach gesetzlichen Vorschriften vernichtet oder gelöscht werden müssen und die nach diesen gesetzlichen Vorschriften nicht ersatzweise den zuständigen öffentlichen Archiven angeboten werden dürfen". Die Formulierung trägt, nebenbei bemerkt, nicht gerade zur Normenklarheit bei: Die Regelung lässt sich sprachlich nämlich sowohl so auslegen, dass 1.) Unterlagen nicht angeboten werden müssen, sofern die Anbietung nicht ausdrücklich vorgeschrieben ist 2.) genau umgekehrt: die Unterlagen angeboten werden müssen, sofern die Anbietung nicht ausdrücklich untersagt ist. Allerdings geht die Gesetzesbegründung wohl deutlich in die erste Richtung, vgl. dazu nochmals PATT, Chancen oder Stolperfallen (wie Anm. 59), sowie die Problematisierung bei PARTSCH, Bundesarchivgesetz (wie Anm. 17), S. 152-155, Rn. 62-67, mit einer summarischen Auflistung von betroffenen Gesetzen ohne explizite Anbietungspflicht.

61 Der Tenor ist eindeutig: „Die Anbietung und Abgabe von Unterlagen nach $\ 5$ Abs. 1 BArchG kann gerade nicht als, Surrogat' der Aktenvernichtung nach $\$ 13$ Abs. 3 BVerfSchG qualifiziert werden. Vielmehr steht - im Gegenteil - die Pflicht und Befugnis des Bundesamtes für Verfassungsschutz zur Vernichtung von Akten von vornherein unter dem Vorbehalt, dass diese Akten nicht nach den Vorschriften des Bundesarchivgesetzes dem Bundesarchiv zur Übernahme anzubieten und zu übergeben sind“.

62 Im Hintergrund steht dabei, dass es laut BVerwG keine Konnexität zwischen Anbietungspflicht und Benutzungsanspruch gibt.

63 Der Verfasser geht davon aus, dass eine „Aktenvernichtung“ mindestens auch die „Löschung" beinhaltet. 
halten werden ${ }^{64}$. Gewiss kann man diese Auslegung rein juristisch auch bestreiten, aber in der praktischen Argumentation zum Zweck der Überlieferungssicherung kommt es darauf im Zweifel nicht an. Aus dem gleichen Grund wird es weiterhin sinnvoll sein, mit dem Surrogat-Argument zu agieren, sofern das Ziel - nämlich die Anbietung sämtlicher Unterlagen ohne Ausnahme - erreicht wird. Gleichwohl stellt das Gericht hier die Konstruktion des Löschungs- und Vernichtungssurrogats in einer Form infrage, die durchaus reflektiert werden sollte: Denn ein „Surrogat" ist eben immer nur ein Ersatz für das, was grundsätzlich gilt - damit lässt sich aber kaum ein Vorrang, hier also der Anbietungspflicht, begründen. Zudem wird indirekt durch Sondervorschriften für bestimmte Unterlagengruppen die Geltung der allgemeinen Anbietungspflicht insgesamt geschwächt, selbst bei bestehenden Surrogatregeln ${ }^{65}$. In dieser Hinsicht ist die Umkehrung zugunsten eines Überwiegens der archivgesetzlichen Anbietungspflicht unabhängig von Archivierungsregelungen in Spezial- oder Datenschutzgesetzen durch das Bundesverwaltungsgericht logischer und zudem archivfreundlicher ${ }^{66}$.

Allerdings löst auch diese Entscheidung nicht alle Probleme: werden nämlich in den Archivgesetzen selbst ${ }^{67}$ oder in entsprechenden Fachgesetzen Unterlagen explizit von der Anbietungspflicht ausgenommen, gibt es keinen - jedenfalls keinen eindeutigen - Vorrang mehr ${ }^{68}$. Weiterhin ist also der Versuch nötig und sinnvoll, solche überlieferungsfeindlichen Vorschriften abzuschaffen oder gar nicht erst in die Archiv- bezie-

$64 \mathrm{Ob}$ es dafür überhaupt eines expliziten Bezugs auf Unterlagen, die bundesrechtlichen Löschungsvorschriften unterliegen, in den Archivgesetzen bedarf (vgl. Reнm, Löschkultur [wie Anm. 59], S. 103), sei dahingestellt.

65 Diese Schwächung wird auch konstatiert von ReHm, Löschkultur (wie Anm. 59), S. 107f., der gleichwohl stark für das Löschungssurrogat als Abhilfe plädiert.

66 Wie problematisch die Auslagerung der Anbietungspflicht in nicht-archivgesetzliche Vorschriften ist, zeigt $\$ 28$ Abs. 1 Satz 1 Nr. 3 in Verbindung mit Satz 2 NDSG: Hier wird für personenbezogene Unterlagen, die Löschungsvorschriften - die Formulierung der „rechtlichen Verpflichtung" ist derart allgemein, dass keineswegs nur Gesetze impliziert sein müssen - unterliegen, das Löschungssurrogat sogar explizit ausgeschlossen. Damit steht diese Vorschrift allerdings in faktischem Gegensatz zum NArchG: Zunächst regelt $\$ 3$ Abs. 1 Satz 2 NArchG, dass auch solches Schriftgut anzubieten ist, „das nach Rechtsvorschriften des Bundes der Geheimhaltung unterliegt, und Schriftgut, das besondere Kategorien personenbezogener Daten im Sinne des Artikels 9 Abs. 1 der Verordnung (EU) 2016/679 (DatenschutzGrundverordnung) enthält“". Weiter regelt der $\$ 3 \mathrm{~b} N A r c h G$ ausdrücklich die Verarbeitung besonderer Kategorien personenbezogener Daten im Archiv, die prinzipiell zulässig ist. Zwar geht das NArchG dem NDSG vor, aber es braucht nicht viel Phantasie sich vorzustellen, dass hier in der praktischen Arbeit durch solche Widersprüche potenziell Probleme entstehen können. Dabei war für den niedersächsischen Landesgesetzgeber bereits bei der Erstfassung des NArchG von $1993 \mathrm{klar}$, dass eine Vernichtung ohne Anbietung nur zulässig ist, wenn ,in bereichsspezifischen Regelungen eine v o r r a n g i g e [Sperrung vom Verfasser] gesetzliche Löschungspflicht festgesetzt wurde“ (Niedersächsischer Landtag, Drucksache 12/4271, hier S. 12) - die bloße Löschungs- oder Vernichtungsregelung ohne Bezug auf die Anbietungspflicht reicht also nicht.

67 Wie etwa im oben erwähnten $\$ 6$ Abs. 2 BArchG Nr. 2.

68 Die in Anm. 59 genannte Literatur diskutiert das Problem u.a. im Hinblick auf Unterlagen, bei denen Landes- oder Kommunalbehörden Bundesrecht ausführen. 
hungsweise Spezialgesetze vordringen zu lassen ${ }^{69}$. Bis dahin liefert obige Entscheidung ein gutes zusätzliches Argument in der alltäglichen Kommunikation mit unsicheren Behördenmitarbeitern.

\section{Fazit: Rechtsprechung und Archivpraxis - Einige Schlussfolgerungen}

Im Ergebnis dieser kleinen Auswahl zeigt sich, dass im Bereich der Benutzung und Überlieferungsbildung zuletzt einige insgesamt die praktische Arbeit der Archive wie auch ihrer Partner (Benutzer, Behörden) erleichternde Entscheidungen ergangen sind. Bedauerliche Ausnahme ist das Thema des Urheberrechts, wo die Lage sich eher verschlechtert hat.

Deutlich geworden sein sollte aber auch: Für die Rechtsprechung kommt es immer auf den Einzelfall an, und auch dieser ist nicht immer oder nur eingeschränkt auf den konkreten Anwendungsfall im Archiv übertragbar. Zudem wird die archivrelevante Rechtsprechung mutmaßlich auch weiterhin eher überschaubar bleiben. Eine Generallösung für die massenhafte Alltagspraxis archivrechtlicher Fragestellungen wird es also auch künftig nicht geben. Weiterhin werden Archive in den Grauzonen abwägen und entscheiden müssen. Andererseits: Wo wenig oder nichts gerichtlich entschieden wurde, gibt es naturgemäß mehr Entscheidungsfreiheit.

Gleichwohl lassen sich aus den besprochenen Entscheidungen auch einige allgemeine Handlungsempfehlungen ableiten beziehungsweise bekannte bestätigen:

1.) Begründen und dokumentieren: (Ermessens-)Entscheidungen sollten stets konkret begründet werden, pauschale Begründungen reichen nicht. Dies ist insbesondere auch für die Nachvollziehbarkeit nach längeren Zeiträumen (Schutzfristen!) wichtig.

2.) Typisierung vor Einzelfallprüfung: Um einen Ausgleich zwischen Rechtskonformität und Umgang mit der Massenproblematik zu finden, sollten Gruppen von Unterlagen mit gleicher archivrechtlicher Einstufung gebildet und diese dann auch gleich behandelt werden.

3.) Rechtsgüterabwägung und Risikoabschätzung verbinden: Dies haben Archive schon immer getan, etwa bei Schutzfristenverkürzungen. Wichtig ist es, nicht unreflektiert einem Grundrecht das Übergewicht zu geben.

4.) Archivrecht instrumentell einsetzen: Niemand kennt archivrelevantes Recht und Rechtsprechung besser als die Archivare selbst - dies sollte ausgenutzt werden, um die Spielräume und Grauzonen im berufsethischen Sinne bestmöglich auszulegen.

5.) Mut zu Entscheidungen: Wegen der vielen rechtlichen Widersprüchlichkeiten und Grauzonen wird es immer Unsicherheiten geben - wichtig ist es daher, überhaupt

69 Dass sich in solchen Vorschriften generell ein komplettes Missverstehen archivischer Zeithorizonte in der Benutzung offenbart, sei nur am Rande erwähnt: spätestens nach ca. 200 Jahren sind sogar postmortale Persönlichkeitsrechte abgelaufen, die Unterlagen aber immer noch und mutmaßlich auf ewig im Archiv. 
Entscheidungen zu treffen und nicht ungeklärte Fragen den Nachfolgern zu überlassen.

6.) Zwischenmenschliche Basis beachten: Angesichts der Grauzonen werden bestimmte Fragen auch künftig nicht allein mittels formaljuristischer Grundlagen, sondern nur mit wechselseitiger Kommunikation und Vertrauen in das Gegenüber gelöst werden können - auch im Internetzeitalter.

Anhang: Zusammenfassung der besprochenen Entscheidungen (alle Entscheidungen sind auch im Internet abrufbar)

\begin{tabular}{|c|c|c|}
\hline Arbeitsfeld & Kernaussage & Gerichtsentscheid \\
\hline \multirow[t]{2}{*}{$\begin{array}{l}\text { Allgemeine Stellung } \\
\text { der Archive }\end{array}$} & $\begin{array}{l}\text { Archive sind im öffentlichen Interesse, sie } \\
\text { dienen der Bildung und Erziehung sowie } \\
\text { der Debatte in der Demokratie }\end{array}$ & $\begin{array}{l}\text { BVerfG, Beschl. v. 06.11.2019- } \\
1 \text { BvR 16/13 } \\
\text { BVerfG, Beschl. v. 06.11.2019- } \\
1 \text { BvR 276/17 }\end{array}$ \\
\hline & $\begin{array}{l}\text { Löschung oder Vernichtung von Berichten } \\
\text { aus Archiven grundsätzlich unzulässig }\end{array}$ & dito \\
\hline \multirow[t]{2}{*}{ Benutzung } & $\begin{array}{l}\text { Kein pauschaler Anspruch auf Nicht-Ver- } \\
\text { offentlichung personenbezogener Daten } \\
\text { auch bei Lebenden }\end{array}$ & $\begin{array}{l}\text { BVerfG, Beschl. v. 06.11.2019- } \\
1 \text { BvR 16/13 } \\
\text { BVerfG, Beschl. v. 06.11.2019- } \\
1 \text { BvR 276/17 }\end{array}$ \\
\hline & $\begin{array}{l}\text { Keine Verpflichtung zu proaktiven Schutz- } \\
\text { maßnahmen und regelmäßigen Überprü- } \\
\text { fungen, ob vormals rechtmäßig veröffent- } \\
\text { lichte personenbezogene Daten immer } \\
\text { noch rechtmäßig online verbreitet werden }\end{array}$ & dito \\
\hline \multirow[t]{2}{*}{$\begin{array}{l}\text { (Interpretation mehre- } \\
\text { rer Paragraphen des } \\
\text { BArchG) }\end{array}$} & $\begin{array}{l}\text { Pauschalisierende Begründungen zur Nut- } \\
\text { zungsversagung aufgrund schutzwürdiger } \\
\text { Belange Dritter unzulässig }\end{array}$ & $\begin{array}{l}\text { Bundesverwaltungsgericht, } \\
\text { Beschl. v. 08.02.2019-20 F 2/17 } \\
\text { Bundesverwaltungsgericht, Urt. } \\
\text { v. 30.01.2019-6 A 1/17 }\end{array}$ \\
\hline & $\begin{array}{l}\text { Das allgemeine Persönlichkeitsrecht endet } \\
\text { mit dem Tod und der allgemeine Achtungs- } \\
\text { anspruch (= postmortales Persönlichkeits- } \\
\text { recht) wird durch die Veröffentlichung } \\
\text { wahrer Tatsachenangaben über einen Ver- } \\
\text { storbenen grundsätzlich nie verletzt }\end{array}$ & dito \\
\hline \multirow[t]{2}{*}{$\begin{array}{l}\text { (Interpretation mehre- } \\
\text { rer Paragraphen des } \\
\text { BArchG) }\end{array}$} & $\begin{array}{l}\text { Allgemeine dreißigjährige Schutzfrist ist je- } \\
\text { weils gesondert für Vorgänge innerhalb von } \\
\text { Akten zu bilden }\end{array}$ & $\begin{array}{l}\text { Bundesverwaltungsgericht, Urt. } \\
\text { v. 11.12.2019-6 C 21/18 }\end{array}$ \\
\hline & $\begin{array}{l}\text { Keine konkludente Zustimmung zur Über- } \\
\text { tragung von Nutzungsrechten bei urheber- } \\
\text { rechtlich geschütztem Schriftgut trotz be- } \\
\text { stehender Anbietungspflicht } \\
\text { Keine Veröffentlichung von urheberrecht- } \\
\text { lich geschütztem Archivgut durch Abgabe } \\
\text { an das Archiv }\end{array}$ & $\begin{array}{l}\text { Bundesverwaltungsgericht, Urt. } \\
\text { v. 26.09.2019-7 C 1/18 } \\
\text { Landgericht Berlin, Beschl. v. } \\
\text { 10.10.2006 - } 16 \text { O 908/06 }\end{array}$ \\
\hline
\end{tabular}




\begin{tabular}{|c|c|c|}
\hline Arbeitsfeld & Kernaussage & Gerichtsentscheid \\
\hline & $\begin{array}{l}\text { Zugänglichmachung von urheberrechtlich } \\
\text { geschütztem Archivgut im Lesesaal greift, } \\
\text { mindestens sofern dieses Schriftgut im Ver- } \\
\text { waltungsverfahren entstanden ist, in das } \\
\text { Veröffentlichungsrecht ein, dies ist nur bei } \\
\text { überwiegendem öffentlichen Interesse zu- } \\
\text { lässig (für in amtlichem Auftrag entstande- } \\
\text { nes Schriftgut kann u.U. eine Veröffentli- } \\
\text { chung durch Einsichtnahme im Lesesaal } \\
\text { verneint werden) }\end{array}$ & $\begin{array}{l}\text { Bundesverwaltungsgericht, Urt. } \\
\text { v. 26.09.2019-7 C 1/18 } \\
\text { Abweichend für im amtlichen } \\
\text { Auftrag entstandene Unterla- } \\
\text { gen: Verwaltungsgericht Magde- } \\
\text { burg, Urt. v. 23.01.2018 - } 6 \text { A } \\
\text { 343/16 MD }\end{array}$ \\
\hline & $\begin{array}{l}\text { Keine Schrankenregelungen außerhalb des } \\
\text { Urheberrechts, daher keine Erlaubnis für } \\
\text { Eingriffe in das Recht des Urhebers durch } \\
\text { das Benutzungsrecht der öffentlichen Ar- } \\
\text { chive }\end{array}$ & $\begin{array}{l}\text { Gerichtshof der Europäischen } \\
\text { Union, Urt. v. 29.07.2019- } \\
\text { C-516/17 }\end{array}$ \\
\hline & $\begin{array}{l}\text { Zitatrecht nach } \int 51 \text { UrhG gilt nur bei ver- } \\
\text { öffentlichten Werken - sofern die Abgabe } \\
\text { ins Archiv nicht als Veröffentlichung anzu- } \\
\text { sehen ist (s.o.): Keine Möglichkeit des Zi- } \\
\text { tierens aus urheberrechtlich geschütztem } \\
\text { Archivgut }\end{array}$ & $\begin{array}{l}\text { Gerichtshof der Europäischen } \\
\text { Union, Urt. v. 29.07.2019-- } \\
\text { C-516/17 (diesbezüglich keine } \\
\text { Abwägung mit anderen Grund- } \\
\text { rechten zugelassen) } \\
\text { Landgericht Berlin, Beschl. v. } \\
\text { 10.10.2006 - } 16 \text { O 908/06 (Ab- } \\
\text { wägung mit anderen Grund- } \\
\text { rechten zugelassen) }\end{array}$ \\
\hline $\begin{array}{l}\text { Erschließung } \\
\text { (Interpretation mehre- } \\
\text { rer Paragraphen des } \\
\text { BArchG) }\end{array}$ & $\begin{array}{l}\text { „Unterlagen“ können auch einzelne } \\
\text { Schriftstücke in Akten sein, „Entstehung“ } \\
\text { bezieht sich auf abgeschlossene Vorgänge } \\
\text { innerhalb von Akten - die allgemeine drei- } \\
\text { Bigjährige Schutzfrist ist daher jeweils ge- } \\
\text { sondert für Vorgänge innerhalb von Akten } \\
\text { zu bilden, deren letzte substanzielle Bear- } \\
\text { beitung ist mit der z.d.A.-Verfügung ab- } \\
\text { geschlossen, eine spätere Wiederaufnahme } \\
\text { führt zu einem neuen Vorgang }\end{array}$ & $\begin{array}{l}\text { Bundesverwaltungsgericht, Urt. } \\
\text { v. 11.12.2019-6 C 21/18 }\end{array}$ \\
\hline \multirow[t]{2}{*}{$\begin{array}{l}\text { Überlieferungsbildung } \\
\text { (Interpretation mehre- } \\
\text { rer Paragraphen des } \\
\text { BArchG) }\end{array}$} & $\begin{array}{l}\text { Keine Sanktionsmöglichkeiten bei Verstoß } \\
\text { gegen die Anbietungspflicht, keine Straf- } \\
\text { barkeit }\end{array}$ & $\begin{array}{l}\text { Bundesverwaltungsgericht, } \\
\text { Beschl. v. 12.09.2017 - 6 A 1/15 } \\
\text { Bundesverwaltungsgericht, Urt. } \\
\text { v. 30.01.2019-6 A } 1 / 17\end{array}$ \\
\hline & $\begin{array}{l}\text { Vorrang der archivgesetzlichen Anbie- } \\
\text { tungspflicht gegenüber allen spezialgesetz- } \\
\text { lichen Löschungs- und Vernichtungsrege- } \\
\text { lungen, soweit archiv- oder spezialgesetz- } \\
\text { lich nicht Ausnahmen von der Anbietungs- } \\
\text { pflicht definiert sind }\end{array}$ & $\begin{array}{l}\text { Bundesverwaltungsgericht, Urt. } \\
\text { v. 11.12.2019-6 C 21/18 }\end{array}$ \\
\hline
\end{tabular}

